\title{
Perinatal outcomes associated with meconium-stained amniotic fluid in Japanese singleton pregnancies
}

\author{
Misao Satomi, Yoshie Hiraizumi, Shunji Suzuki
}

Department of Obstetrics and Gynecology, Japanese Red Cross Katsushika Maternity Hospital, Tokyo, Japan. Email: czg83542@mopera.ne.jp

Received 3 May 2011; revised 31 May 2011; accepted 9 June 2011.

\begin{abstract}
Introduction: We examined the perinatal outcomes in Japanese singleton pregnancies associated with meconium-stained amniotic fluid (MSAF) in relation to gestational age at delivery. Methods: We reviewed the obstetric records of all Japanese singleton deliveries after 22 weeks' gestation managed at Japanese Red Cross Katsushika Maternity Hospital between 2002 and 2008 ( $n=11,249)$. Results: The incidence of MSAF in the whole singleton pregnancies was $13 \%$. The incidence of MSAF at preterm, term and postterm were $9.1 \%, 13 \%$ and $48 \%$, respectively. The incidence of intrauterine fetal death, low Apgar score and low umbilical artery $\mathrm{pH}$ at delivery in cases with MSAF were significantly higher than those without MSAF in various gestational ages at delivery. Conclusion: Obstetric management should be affected by meconium in the amniotic fluid.
\end{abstract}

Keywords: Meconium-Stained Amniotic Fluid; Perinatal Outcome; Preterm; Term; Postterm

\section{INTRODUCTION}

Meconium-stained amniotic fluid (MSAF) has been reported to be associated with an obstetric hazard and significantly increase risks of adverse neonatal outcomes at term and preterm [1-7]. Although overall risk of adverse outcome in MSAF has been reported to be low [2], MSAF is suggested to signify underlying acute or chronic fetal hypoxia [1-7]. Recently, MSAF rates have been reported to be different among races and across gestational age [2]. For example, Balchin et al. [2] observed that the incidence of MSAF in South Asian is higher than that in whites (Crude odds ratio 3.31, 95\% confidence interval $1.3-8.3, p<0.01$ by $x^{2}$ test); however there have not been well documented in MSAF in Japanese populations. In this study, we examined the perinatal outcomes in Japanese singleton pregnancies associated with MSAF in relation to gestational age at delivery.

\section{METHODS}

The protocol for this study was approved by the Ethics Committee of the Japanese Red Cross Katsushika Maternity Hospital. In addition, informed consent concerning analysis from a retrospective database was obtained from each subject.

We reviewed the obstetric records of all Japanese singleton deliveries after 22 weeks' gestation managed at Japanese Red Cross Katsushika Maternity Hospital between 2002 and $2008(\mathrm{n}=11,249)$. The gestational age of the pregnancies were established by ultrasonographic examination of the fetal crown-rump length at 9-11 weeks' gestation. In all cases of intrauterine fetal death (IUFD), survival was checked within 2 weeks before the period of the IUFD diagnosis. The presence of MSAF was diagnosed clinically during delivery. The characteristics of perinatal outcomes such as IUFD, neonatal Apgar score at 1 and 5 minutes and umbilical artery $\mathrm{pH}$ were extracted from patient charts. In this study, the subjects were divided into 5 groups by gestational age at delivery as follows: those delivered at 22 - 28, $29-32$, 33 - 36, 37 - 40 and 41 - 43 weeks' gestation.

Cases and controls were compared by $x^{2}$ test for categorical variables. Odds ratios (ORs) and 95\% confidence intervals (CIs) were also calculated. Differences with $P$ $<0.05$ were considered significant.

\section{RESULTS}

Table 1 shows the incidence of MSAF in the Japanese singleton pregnancies by gestational age at delivery. The incidence of MSAF in the whole singleton pregnancies was $13 \%(1,409 / 11,249)$. The incidence of MSAF at preterm (22 - 36 weeks), term (37 - 41 weeks) and postterm (42 - 43 weeks) were 9.1\% (73/804, $p<0.01$ vs. term, OR 0.70, 95\% CI 0.54 - 0.89), 13\% (1,297/10,363) and $48 \%(39 / 82, p<0.01$ vs. term, OR $6.34,95 \%$ CI 4.1 - 9.8), respectively. 
Table 2 shows the incidence of MSAF in the 5 periods of deliveries in the Japanese singleton pregnancies. As shown in Table 2, the incidence of MSAF at 33 - 36 weeks was significantly lower than that at $37-40$ weeks $(p<0.01)$, however the incidence of MSAF at $22-31$ weeks was not different significantly from that at $37-40$ weeks $(p=0.24)$. The incidence of MSAF at $41-43$ weeks was significantly higher than that at $37-40$ weeks $(p<0.01)$. Therefore, there was a 'J-shaped' relationship between MSAF and advancing gestational age, with a nadir at 33 - 36 weeks' gestation.

Table 3 shows the perinatal outcomes in the 5 periods of deliveries in the Japanese singleton pregnancies. In total, the incidence of IUFD, low Apgar score and low umbilical artery $\mathrm{pH}$ at delivery in cases with MSAF were significantly higher than those without $\operatorname{MSAF}(p<$
0.01). The incidence of IUFD in cases with MSAF was significantly higher than that without MSAF at $29-32$ $(p=0.03)$ and $33-36$ weeks' gestation $(p<0.01)$. The incidence of neonatal low Apgar score at 1 minute in cases with MSAF was significantly higher than that without MSAF at $33-36(p<0.01), 37-0(p<0.01)$ and 41 - 43 weeks' gestation $(p<0.01)$. The incidence of neonatal low Apgar score at 5 minute in cases with MSAF was significantly higher than that without MSAF at 22 - 28 ( $p=0.02), 33-36(p<0.01), 37-40(p<0.01)$ and 41 - 43 weeks' gestation $(p<0.01)$. In addition, the incidence of low umbilical artery $\mathrm{pH}$ in cases with MSAF was significantly higher than that without MSAF at $22-28(p=0.02), 33-36(p<0.01), 37-40(p=0.03)$ and $41-43$ weeks' gestation $(p<0.01)$.

Table 1. The incidence of msaf in the japanese singleton pregnancies by gestational age at delivery.

\begin{tabular}{|c|c|c|}
\hline Gestational age at delivery (weeks) & Number of delivery & Meconium-stained amniotic fluid \\
\hline 22 & 7 & $2(29 \%)$ \\
\hline 23 & 5 & $1(20 \%)$ \\
\hline 24 & 7 & $1(14 \%)$ \\
\hline 25 & 13 & $4(31 \%)$ \\
\hline 26 & 11 & $1(9.1 \%)$ \\
\hline 27 & 22 & $4(18 \%)$ \\
\hline 28 & 25 & $1(4.0 \%)$ \\
\hline 29 & 47 & $3(6.4 \%)$ \\
\hline 30 & 46 & $6(13 \%)$ \\
\hline 31 & 49 & $6(12 \%)$ \\
\hline 32 & 87 & $10(11 \%)$ \\
\hline 33 & 98 & $8(8.2 \%)$ \\
\hline 34 & 155 & $9(5.8 \%)$ \\
\hline 35 & 143 & $8(5.6 \%)$ \\
\hline 36 & 289 & $9(3.1 \%)$ \\
\hline 37 & 943 & $31(3.3 \%)$ \\
\hline 38 & 1,873 & $127(6.8 \%)$ \\
\hline 39 & 2,902 & $327(11 \%)$ \\
\hline 40 & 2,877 & $484(17 \%)$ \\
\hline 41 & 1,568 & $328(21 \%)$ \\
\hline 42 & 78 & $37(47 \%)$ \\
\hline 43 & 4 & $2(50 \%)$ \\
\hline Total & 11,249 & $1,409(13 \%)$ \\
\hline
\end{tabular}

Values are expressed as number (\%). $P$ values by $X^{2}$ test. 
Table 2. The incidence of MSAF in the 5 periods of delivery in the Japanese singleton pregnancies.

\begin{tabular}{cccccc}
\hline Gestational age at delivery & Number of delivery & Meconium-stained amniotic fluid & P-value & Crude OR & $95 \%$ CI \\
\hline $22-28$ weeks & 90 & $14(16 \%)$ & 0.2 & 1.45 & $0.82-2.6$ \\
$29-32$ weeks & 229 & $25(11 \%)$ & 0.87 & 0.96 & $0.63-1.5$ \\
$33-36$ weeks & 685 & $34(5.2 \%)$ & $<0.01$ & 0.41 & $0.29-0.58$ \\
$37-40$ weeks* & 8,595 & $969(11 \%)$ & - & 1 & 2.25 \\
$41-43$ weeks & 1,650 & $367(22 \%)$ & $<0.01$ & $2.0-1.6$ \\
Total & 11,249 & $1,409(13 \%)$ & & & \\
\hline
\end{tabular}

*Reference group. Values are expressed as number (\%). P values by X2 test. OR, odds ratio; $95 \%$ CI, $95 \%$ confidence interval; IUFD, intrauterine fetal death.

Table 3. The perinatal outcomes in the 5 periods of delivery in the Japanese singleton pregnancies.

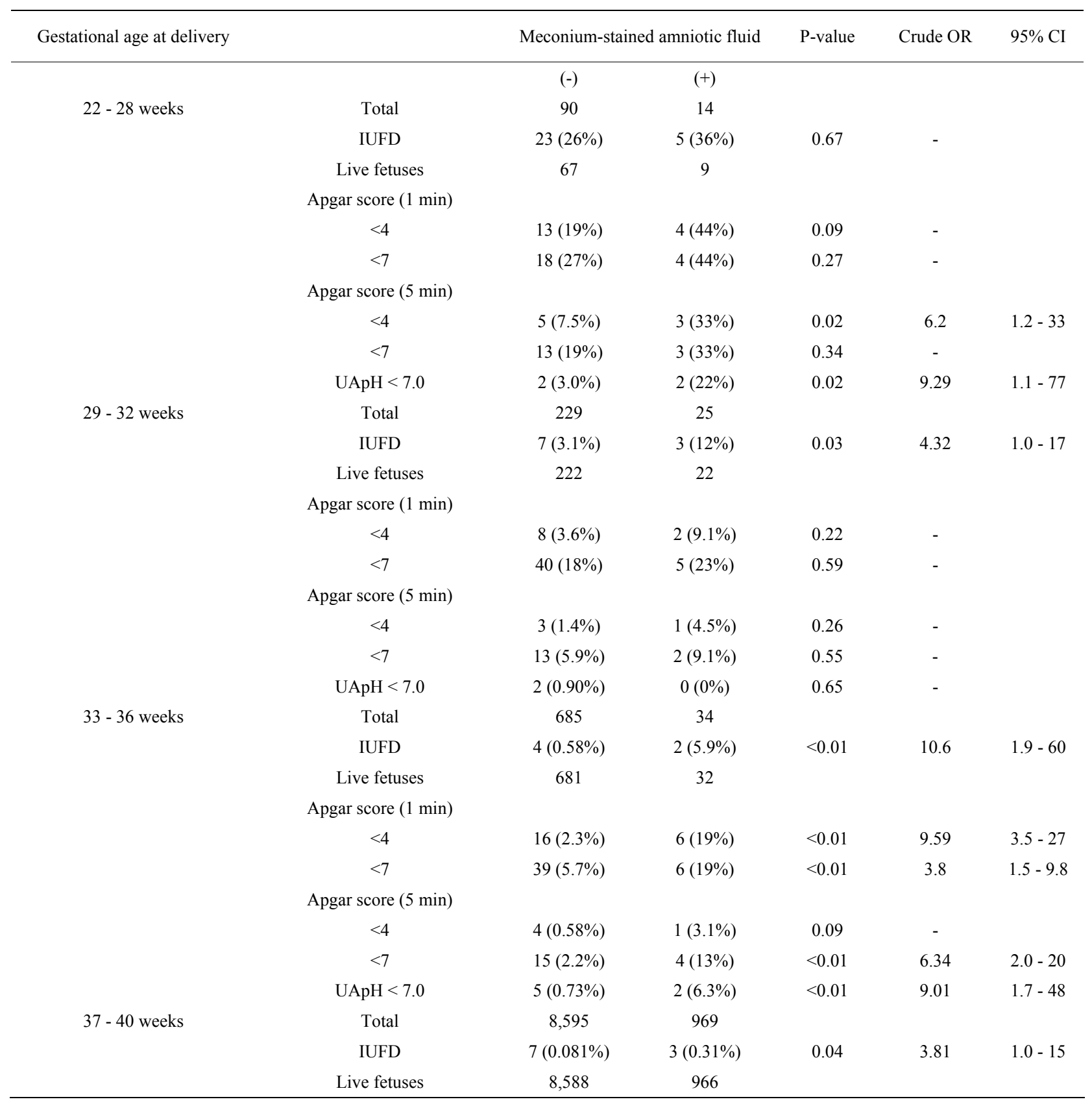




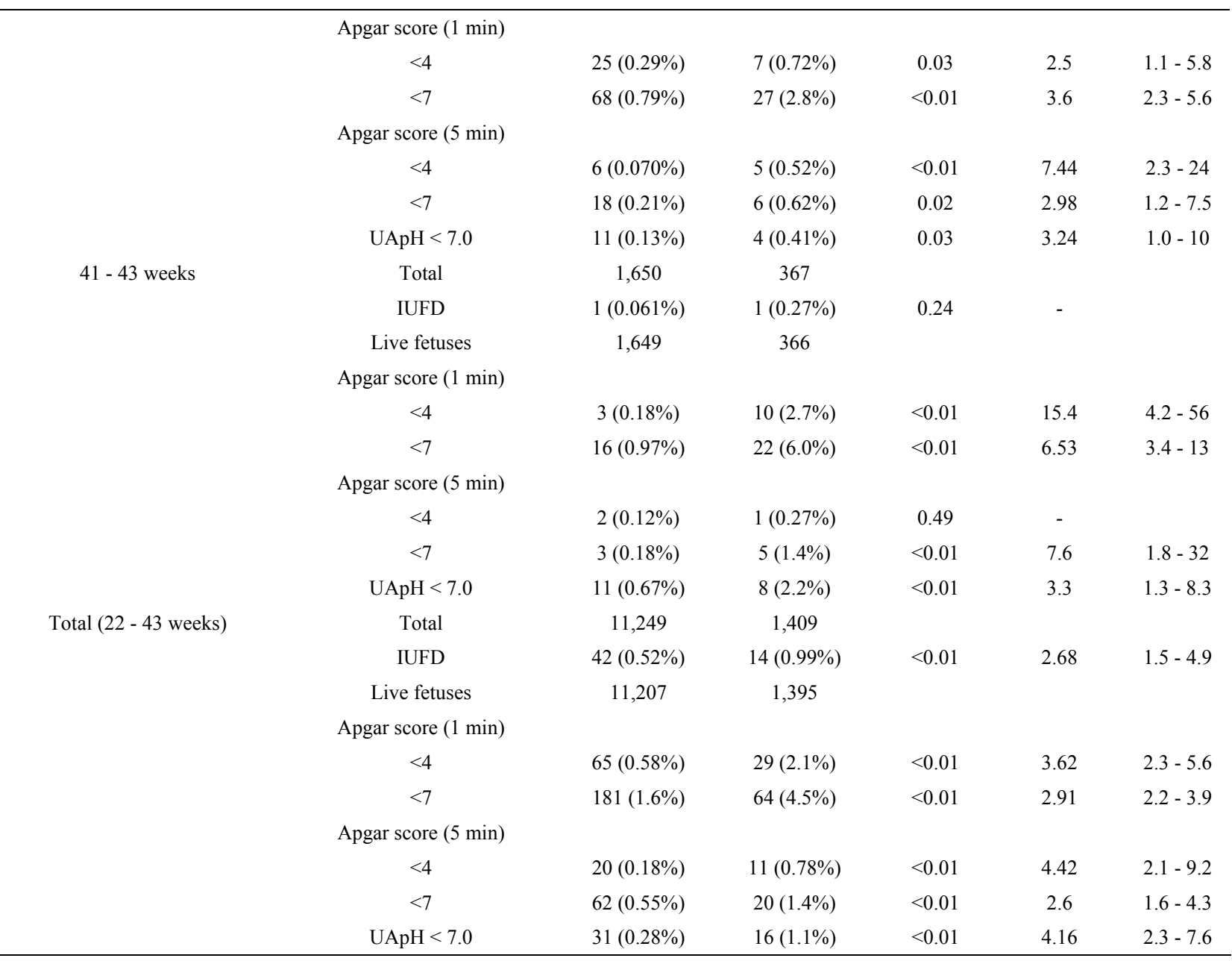

Values are expressed as number (\%). P values by $X^{2}$ test. OR, odds ratio; 95\% CI, 95\% confidence interval; IUFD, intrauterine fetal death.

\section{DISCUSSION}

The relationship between the presence of MSAF and increased odds for birth asphyxia and neonatal mortality is well established in preterm, term and postterm infants [1-7]. We also found that in the infants with MSAF, the prevalence of IUFD and/or neonatal asphyxia (low Apgar scores and/or low umbilical artery $\mathrm{pH}$ ) was increased compared with those without MSAF in Japanese singleton pregnancies in various gestational age at delivery. Therefore, obstetric management should be affected by meconium in the amniotic fluid in all periods of pregnancy beyond 22 weeks' gestation.

In this study, there was a "J-shaped" relationship between MSAF and advancing gestational age, with a nadir at late-preterm (33 - 36 weeks' gestation); because MSAF has been suggested to be mainly associated with fetal gastrointestinal maturity rather than hypoxia under hormonal and neural control $[1,2,6]$. This 'J-shaped' tendency seems to be similar to some previous studies $[2,6]$; however the period of nadir in this study seems to be later than those in these previous studies. For example, the incidence of preterm MSAF at $<33$ weeks observed by Tybulewicz et al. [6] and Balchin et al. [2] were only $4.3 \%$ and $5.3 \%$, respectively; however it was $12 \%$ in the current study. The small sample size and/or the racial differences may be possible reasons leading to the differences. One of other possible reasons may be that the current data included the cases of IUFD. In this study, for example, the incidence of MSAF in live births at $<33$ weeks was $9.7 \%$. Some serious perinatal complications such as cerebral palsy and/or severe intraventricular hemorrhage have been reported to be more common in infants with preterm MSAF [5,6]. Therefore, it may be reasonable that the incidence of MSAF increased in this study including IUFD as serious complication, because over half of IUFD has been reported to occur during the premature period [8]. Otherwise, very premature delivery itself can be indicated as "serious complication" equivalent to IUFD. Therefore, further prospective examination may be needed to clarify the relation between 
MSAF and perinatal outcomes at very preterm.

At 41 - 43 weeks' gestation, on the other hand, the incidence of low Apgar score and low umbilical artery $\mathrm{pH}$ in cases with MSAF were also significantly higher than those without MSAF. Postterm gestation itself has been suggested to be associated with the increased risk of perinatal mortality [9]. The higher rate of perinatal morbidity at postterm gestation may be due to hypoxia/ acidemia associated with "relative placental insufficiency" where the placenta can no longer keep up the demands of the fetus [10]. These conditions may be also associated with the presence of MSAF. In addition, when MSAF is superimposed on fetal acidemia, there is an increased risk of meconium aspiration syndrome [4]. Therefore, the greater risk of adverse neonatal outcomes at $\geq 41$ weeks in the current study support these previous suggestions especially in cases with MSAF $[4,10]$.

In conclusion, obstetric management should be affected by meconium in the amniotic fluid in various gestational ages at delivery. Therefore, management requires awareness of this potential risk, appropriate intrapartum care and a combined obstetric-neonatal approach in cases with MSAF.

\section{REFERENCES}

[1] Ash, A.K. (2000) Managing patients with meconiumstained amniotic fluid. Hospital Medicine, 61, 844-848.

[2] Balchin, I., Whittaker, J.C., Lamont, R.F. and Steer, P.J. (2011) Maternal and fetal characteristics associated with meconium-stained amniotic fluid. Obstetrics \& Gynecology, 117, 828-835.

doi:10.1097/AOG.0b013e3182117a26
[3] Natah, L., Leveno, K.J., Carmody III, T.J., Kelly, M.A. and Sherman, M.L. (1994) Meconium: A 1990s perspective on an old obstetric hazard. Obstetrics \& Gynecology, 83, 329-332.

[4] Ramin, K.D., Leveno, K.J., Kelly, M.A. and Carmody, T.J. (1996) Amniotic fluid meconium: A fetal environmental hazard. Obstetrics \& Gynecology, 87, 181-184. doi:10.1016/0029-7844(95)00403-3

[5] Spinillo, A., Fazzi, E., Capuzzo, E., Stronati, M., Piazzi, G. and Ferrari, A. (1997) Meconium-stained amniotic fluid and risk for cerebral palsy in preterm infants. $\mathrm{Ob}$ stetrics \& Gynecology, 90, 519-523. doi:10.1016/S0029-7844(97)00308-6

[6] Tybulewicz, A.T., Clegg, S.K., Fonfe, G.J. and Stenson, B.J. (2004) Preterm meconium staining of the amniotic fluid: associated findings and risk of adverse clinical outcome. Archives of Disease in Childhood-Fetal \& Neonatal Edition, 89, F328-F330. doi:10.1136/adc.2002.021949

[7] Ziadeh, S.M. and Sunna, E. (2000) Obstetric and perinatal outcome of pregnancies with term labour and meconium-stained amniotic fluid. Archives of Gynecology and Obstetrics, 264, 84-87. doi: $10.1007 / \mathrm{s} 004040000088$

[8] Gardosi, J., Mul, T., Mongelli, M. and Fagan, D. (1998) Analysis of birthweight and gestational age in antepartum stillbirths. British Journal of Obstetrics and Gynecology, 105, 524-530. doi:10.1111/j.1471-0528.1998.tb10153.x

[9] Suzuki, S. (2009) Post-term pregnancy as a risk factor for intrapartum fetal distress. Journal of Perinatal Medicine, 37, 420-421. doi:10.1515/JPM.2009.055

[10] Volherr, H. (1975) Placental insufficiency in relation to postterm pregnancy and fetal postmaturity: Evaluation of fetoplacental function; management of the postterm gravida. American Journal of Obstetrics \& Gynecology, 123, 67-103. 